\title{
SPL Combination Product Type Terminology
}

National Cancer Institute

\section{Source}

National Cancer Institute. SPL Combination Product Type Terminology. NCI Thesaurus.

Code C102833.

Terminology used for representation of the type of part 3 combination in the framework

of the Structured Product Labeling documents. 